\title{
Identification of genes for salt tolerance and yield-related traits in rice plants grown hydroponically and under saline field conditions by genome-wide association study
}

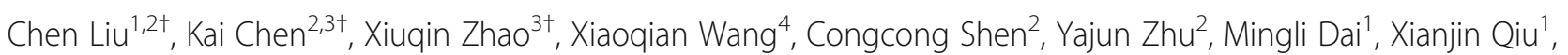
Rongwei Yang ${ }^{1}$, Danying Xing ${ }^{1}$, Yunlong Pang ${ }^{4^{*}}$ and Jianlong $X u^{3^{*}}$ (D)

\begin{abstract}
Background: Soil salinity is one of the main environmental conditions that affects rice production. Identifying the genetic loci that affect rice salt tolerance (ST)-related traits at the seedling stage, especially under saline field conditions, is crucial for ST rice breeding by pyramiding ST genes that act at different developmental stages.

Results: Large phenotypic variations were observed in 708 rice accessions, and yield and its related traits were considerably limited when exposed to salt stress. In a genome-wide association study (GWAS), 2255 marker-trait association signals were detected for all measured traits, and the significant SNPs were distributed in 903 genes. Of these, 43 genes processed same functional annotation, and the gene ontology terms "biological processes" and "molecular function" with the known genes responsive to salt stress in rice. Further haplotype analysis detected 15 promising candidates significantly associated with the target traits, including five known genes and 10 novel genes. We identified seven accessions carrying favorable haplotypes of four genes significantly associated with grain yield that performed well under saline stress conditions.

Conclusions: Using high density SNPs within genes to conduct GWAS is an effective way to identify candidate genes for salt tolerance in rice. Five known genes (OsMYB6, OsGAMYB, OsHKT1;4, OsCTR3, and OsSUT1) and two newly identified genes (LOC_Os02g49700, LOC_Os03g28300) significantly associated with grain yield and its related traits under saline stress conditions were identified. These promising candidates provide valuable resources for validating potential ST-related genes and will facilitate rice breeding for salt tolerance through marker-assisted selection.
\end{abstract}

Keywords: Rice, Salt tolerance, Genome-wide association study (GWAS), Candidate gene, Quantitative trait locus/ loci (QTL)

\footnotetext{
* Correspondence: xujlcaas@126.com; y.pang@sdau.edu.cn

${ }^{+}$Chen Liu, Kai Chen and Xiuqin Zhao equally contributed to the work.

${ }^{3}$ Institute of Crop Sciences, Chinese Academy of Agricultural Sciences, Beijing

100081, China

${ }^{4}$ State Key Laboratory of Crop Biology, College of Agronomy, Shandong

Agricultural University, Taian 271018, China

Full list of author information is available at the end of the article
} 


\section{Background}

Rice (Oryza sativa L.), one of the most important crops, is a staple food in the diet of more than half of the world's population. Rice production is adversely influenced by numerous biotic and abiotic stresses including diseases and pests, cold, drought, flooding, heat, and salinity. Soil salinity is a common abiotic stress in many rice production areas that causes serious reductions in productivity. Globally, more than 930 million ha of land are thought to be affected by salinity, with China having over 100 million ha ( $\mathrm{Hu}$ et al. 2012). Due to current salinity levels and other soil-related problems, millions of hectares in the humid regions of South and Southeast Asia where rice is the main food crop are either left uncultivated, or rice is grown with extremely low yields. Increasing soil salinization is one of the main obstacles affecting rice production. Developing rice varieties with good salt tolerance (ST) and high yield is considered to be one of the most economic options to utilize salinized soil for rice production (Blumwald and Grover 2006).

Generally, rice is considered to be moderately sensitive to salinity (Ismail and Thomson 2011). When the electrical conductivity of soil saturated extract is $5-6 \mathrm{ds} / \mathrm{m}$, the growth of salt-sensitive rice would be negatively affected and the yield would be reduced by half (Abdullah et al. 2001; Fageria 1985; Yeo et al. 1990). However, different rice genotypes vary considerably in their ST characteristics (Gregorio et al. 2002), which provides the basis of breeding for ST rice varieties. To decipher the genetic architecture of ST in rice, the quantitative trait loci (QTL) mapping approach has been used by many researchers to identify QTL at various growth stages including germination (Cheng et al. 2015; Wang et al. 2011), seedling (Cheng et al. 2011; Wang et al. 2012; Zheng et al. 2015) and the reproductive stages (Chai et al. 2013; Hossain et al. 2015; Kumar et al. 2015).

The seedling stage is one of the most vulnerable growth stages to salt stress; tolerance can be measured fairly easily, and many genes affecting ST in seedlings have been identified. SKC1 was the first ST-related gene to be cloned in rice via map-based cloning (Ren et al. 2005). Through the use of mutants and gene overexpression and knockout, approximately 200 genes have been shown to be involved in salt stress at the seedling stage (Molla et al. 2015). ST at the reproductive stage is necessary for high yield under saline conditions. A large number of QTL for ST-related traits at the reproductive stage have been identified through linkage mapping and association analysis. For example, in an $\mathrm{F}_{2}$ population derived from a cross between 'Cheriviruppu' (salt-tolerant) and 'Pusa Basmati 1' (salt-sensitive), Hossain et al. (2015) detected 16 QTL affecting plant height, tiller number, panicle length, grain yield per plant, biomass, pollen fertility, and $\mathrm{Na}^{+}$content and the $\mathrm{K}^{+} / \mathrm{Na}^{+}$ratio in the flag leaf measured under stress conditions at the reproductive stage. Kumar et al. (2015) conducted an association study using 220 Xian (indica) lines genotyped using a custom-designed $6 \mathrm{~K}$ single nucleotide polymorphisms (SNPs) array and identified 64 SNPs significantly associated with the $\mathrm{K}^{+} / \mathrm{Na}^{+}$ratio as well as other agronomic traits including filled grain number, productive tiller number, seed setting rate, stress susceptibility index of the seed setting rate, and grain yield observed at the reproductive stage under salt stress. However, to the best of our knowledge, no QTL for ST at the reproductive stage have been fine-mapped or cloned.

Over the past several years, association studies using high density genome-wide SNP markers detected by next-generation sequencing have been shown to be a powerful strategy to mine candidate genes associated with traits of interest. Recently, the 3000 rice genomes project (3 K RGP) used Illumina next-generation sequencing of a core collection of 3024 rice accessions from 89 countries to generate sequence data with high coverage $(\sim 94 \%)$ and mapping rate $(\sim 92.5 \%)$ and to construct a high-density SNP database providing genotypic data for genome-wide association studies (GWAS) of agronomic traits in rice (Wang et al. 2018).

In this study, 708 rice accessions selected from the 3024 rice genomes sequenced by the $3 \mathrm{~K}$ RGP were used to conduct an association study to identify genetic loci for ST. For this analysis, we used 1,101,404 SNPs within genes filtered from the $3 \mathrm{~K}$ RGP $32 \mathrm{M}$ SNP raw dataset in the Rice SNP-Seek Database (Alexandrov et al. 2015). The goals of this study were to: 1) identify genes associated with rice ST at the seedling stage, 2) detect genes associated with yield and its related traits under saline field conditions, and 3) analyze promising gene candidates that are potentially involved in tolerance to salt stress and identify favorable haplotypes. This information will be useful for further mining of functional genes for rice ST, and for the markerassisted breeding of ST varieties suitable for cultivation in salinized paddies.

\section{Materials and methods}

\section{Plant materials}

In total, 708 rice accessions selected from the $3 \mathrm{~K}$ RGP were used in this study, which included 400 Xian (indica), 247 Geng (japonica), 39 intermediate types, 16 Aus, and six Basmati varieties (Wang et al. 2018). The geographical origins of these accessions included 77 countries or regions with most of accessions originating from China (205), followed by the Philippines (70), India (56), Japan (38), Italy (25), and the United States (24). For other countries or regions, the number of accessions were $<20$ (Additional file 1: Table S1). 
Evaluation of ST at the seedling stage under hydroponic conditions

An experiment to evaluate ST at the seedling stage was carried out in a greenhouse at the Institute of Crop Sciences, Chinese Academy of Agricultural Sciences (CAAS), Beijing. Plastic containers were prepared for the screening, and styrofoam seedling float trays were used that fit inside the plastic containers. Each styrofoam tray consists of an array of $10 \times 13$ holes with a styrofoam nylon net attached to the bottom. The rice seeds were surface sterilized with $5 \%$ sodium hypochlorite solution for $20 \mathrm{~min}$ and rinsed well with distilled water. Seeds were soaked in water for $48 \mathrm{~h}$ at $30^{\circ} \mathrm{C}$ to allow them to germinate. A single healthy germinated seed was placed in each hole, and 10 seeds were sown for each accession. During the first two days, the seedlings were watered with tap water, after which it was changed to Yoshida culture solution (Yoshida et al. 1976). When the seedlings were at the three-leaf stage two weeks after seeding, $\mathrm{NaCl}$ was added to the culture solution at concentrations up to $140 \mathrm{mM}$. The $\mathrm{pH}$ of the culture solution was adjusted daily to 5.0 by adding either $\mathrm{NaOH}$ or $\mathrm{HCl}$, and the solution was renewed every five days. The seedlings were scored based on visual symptoms of salt stress injury (SSI) when the susceptible check IR29 was given a score of 9 as described in the modified Standard Evaluation System (IRRI 2013). Seedling survival days (SSD) was recorded on a daily basis after the first plant had died.

\section{Yield trials under saline field conditions}

The association panel was evaluated for grain yield and related traits in replicated field trials conducted under salt stress condition near the coastline at Sanmen $\left(18.3^{\circ} \mathrm{N}, 109.3^{\circ} \mathrm{E}\right)$ in Zhejiang Province, China in 2016 (Y16) and 2017 (Y17). Field trials were conducted following a randomized complete block design with two replicates in 2016 and three replicates in 2017. Twenty germinated seeds were directly and evenly sown in a non-salt-stress normal field in $50 \mathrm{~cm}$-long rows with 25 $\mathrm{cm}$ spacing between rows. Salt stress was introduced after the 2-leaf stage and maintained up to maturity by irrigating with $0.5 \%$ salt water made by proportionally mixing river and sea water. Seedling numbers $(\mathrm{SN})$ in each plot were counted one month after salt application. Heading date (HD, in days) was visually recorded in Y17 for each plot when $\sim 50 \%$ of plants in a plot had headed. At maturity, total panicle number (PN) was recorded and five uniform main panicles in the middle of each plot were sampled to measure total spikelet number (TSN), filled grain number (FGN), seed setting rate (SSR, \%), and thousand-grain weight (TGW, in g). The remaining seeds in each plot were harvested and bulked to measure grain yield per plot (GY, in g).

\section{Statistical phenotypic analyses}

Phenotypic analyses were conducted using linear mixed models. For single-site analysis, accession (genotype) was regarded as a fixed effect and replicate as random effect. The best linear unbiased estimates (BLUE) of the accessions were obtained. For multi-site analysis, all effects including accession (genotype), environment, and replicate within environment were regarded as random to estimate variance components. Narrow-sense heritability $\left(\mathrm{h}^{2}\right)$ based on genotypic means was computed using the estimated variance components as $\mathrm{V}_{\mathrm{G}} /\left(\mathrm{V}_{\mathrm{G}}+\mathrm{V}_{\mathrm{GEI}} / \mathrm{s}+\right.$ $\mathrm{V}_{\mathrm{e}} / \mathrm{sr}$ ). Where $\mathrm{V}_{\mathrm{G}}, \mathrm{V}_{\mathrm{GEI}}$, and $\mathrm{V}_{\mathrm{e}}$ are the variance due to genotype, genotype by environment interaction (GEI), and residual error, respectively; $\mathrm{s}$ is the number of environments and $\mathrm{r}$ is the number of replicates. All analyses were conducted using the PBTools package developed by IRRI (bbi.irri.org). Phenotypic correlations were computed using the BLUE with the "rcorr" function implemented in the R package Hmisc (Harrell 2015).

\section{Manipulating genotype data from the $3 \mathrm{~K}$ RGP}

The $32 \mathrm{M}$ SNPs raw data of the association panel were extracted from the $3 \mathrm{~K}$ RGP in the Rice SNP-Seek Database (http://snp-seek.irri.org/). Genotype cleaning was carried out according to the following steps: 1) for SNPs with more than two allele types, only the top two major alleles were retained, and the other allele types were regarded as missing data; 2) SNPs with minor allele frequency (MAF) $<0.05$ and missing rate $>20 \%$ were removed; 3) a subset of SNPs were randomly sampled for analyzing population structure by calculating kinship and performing a principle component analysis (PCA) using the GAPIT R package (version 3.0); 4) based on gene functional annotations of the reference Geng ( $j a-$ ponica) 'Nipponbare' genome IRGSP-1.0 from the Rice Genome Annotation Project (http://rice.plantbiology. msu.edu/), SNPs within annotated genes were extracted to perform a GWAS.

\section{GWAS using gene-based SNPs}

We carried out GWAS to detect marker-trait associations for all measured traits using SNPs within annotated genes using the GAPIT (version 3.0) $\mathrm{R}$ package that uses EMMA (efficient mixed model association), CMLM (compressed mixed linear model), and P3D (population parameters previously determined) to speed up computation time and optimize statistical performance in the GWAS. The mixed linear model (MLM) allowed correction for cryptic relatedness and other fixed effects using kinship matrix (K) and population stratification through principle components $(\mathrm{P})$. The Bonferroni multiple testing correction was applied to identify significant markers. Significant SNPs affecting the investigated traits were claimed when the test 
statistics reached $P<1.0 \times 10^{-4}$. Individual marker effects were extracted from the model and visualized in a Manhattan plot prepared with the $\mathrm{R}$ package qqman (Turner 2014).

\section{Identifying promising candidate genes for salt tolerance}

To detect promising candidate genes for ST in rice, the identified associated genes were compared with genes that are known to be responsive to salt stress from searches of the funRiceGenes (https://funricegenes. github.io/) and QTARO databases (http://qtaro.abr. affrc.go.jp). Gene ontology (GO) analysis was carried out using 'AgriGO v2' (http://bioinfo.cau.edu.cn/ agriGO/analysis.php). Gene functional annotations were extracted from the MSU Rice Genome Annotation Project Release 7. For genes showing the same functional annotation and GO terms with known genes, haplotype analysis was further carried out and the differences in target traits between/among major haplotypes (with more than 10 accessions) were tested by ANOVA. The genes with significance level $P<0.001$ were considered to be promising ST candidates.

\section{Results}

\section{Phenotypic variation and correlations}

ST at the seedling stage varied widely among the accessions, with SSI ranging from 1 to 9 and SSD ranging from 11 to 23 days. Overall, the Geng (japonica) subspecies had lower values for SSI and higher values for SSD compared with the Xian (indica) subspecies. The mean SSI (SSD) was 3.3 (19.0) for the Geng subspecies but 5.2 (16.5) for the Xian subspecies, which indicated that Geng-type varieties are more tolerant to salt stress than are Xian varieties (Fig. 1 a). Large variations in all the investigated agronomic traits were observed in the rice association panel grown under salt stress conditions in the two field years. Salt stress seriously limits growth and grain yield in rice. The performance in Y16 was slightly worse than it was in Y17, suggesting that salt stress experienced in Y16 was more severe (Fig. 1 b). GY was significantly positively correlated with its components except TGW. The trait correlation coefficients ( $r$ ) between the two years ranged from 0.51 for TGW to 0.79 for TSN (Fig. 1 c). Variance component analysis of the traits showed that $\mathrm{V}_{\mathrm{G}}$ was larger than the other components, indicating that the phenotypic variances were mainly determined by genotype. The estimated heritability $\left(h^{2}\right)$ varied from 0.59 for TGW to 0.88 for TSN (Table 1$)$.

\section{Distribution of SNPs}

After removing SNPs with MAF $<0.05$ and missing data rate $>20 \%$, there wer $3,455,952$ SNPs remaining; the number of SNPs per chromosome ranged from 212,
314 (chromosome 9) to 404,439 (chromosome 1). We randomly sampled $10 \%(345,600)$ of the SNPs for the kinship and PCA analyses. The average marker spacing over the whole genome was $1079 \mathrm{bp}$ with a range of $933 \mathrm{bp}$ on chromosome 10 to $1244 \mathrm{bp}$ on chromosome 5. In total, we identified $1,101,404$ SNPs in 44,332 annotated genes. Each gene contained around 25 SNPs on average ranging from at least one SNP to as many as 649 SNPs (Table 2).

\section{GWAS using gene-based SNPs}

The kinship and PCA analyses showed two distinct subpopulations in the association panel (Fig. 2). One group was comprised of 265 accessions mainly belonging to the Geng subspecies, and the other group was comprised of 443 accessions mainly belonging to the Xian subspecies (Additional file 1: Table S1). Thus, to partially eliminate the false positives induced by population structure, we performed a GWAS for each group separately. The Manhattan plots and QQ plots of the associations for each trait are shown in Additional file 1: Figure. S2A-I.

At the seedling stage, 321 marker-trait associations were detected that included 108 associations identified in the Geng group and 213 associations in the Xian group, with none of these associations detected in both groups. The SNP markers were distributed in 142 gene regions which included 188 SNPs in 95 genes associated with SSI, and 133 SNPs in 63 genes for SSD. Among them, 49 SNPs in 16 genes were associated with the two traits simultaneously (Table 3 and Additional file 1: Table S2).

Under saline field conditions in 2016, 1085 markertrait associations for all seven measured traits were detected including 169 associations identified in the Geng group and 916 identified in the Xian group. None of the associations were detected in both groups. These SNP markers were distributed in 415 genes which included 67 SNPs in 50 genes associated with SN, 27 SNPs in 20 genes associated with GY, 230 SNPs in 96 genes associated with PN, 269 SNPs in 104 genes associated with TSN, 288 SNPs in 93 genes associated with FGN, 179 SNPs in 63 genes associated with SSR, and 25 SNPs in 22 genes associated with TGW (Table 3 and Additional file 1: Table S2).

Under saline field conditions in 2017, 849 marker-trait associations for all measured traits were detected including 350 associations identified in the Geng group and 499 identified in the Xian group. These SNP markers were distributed in 403 genes which included 186 SNPs in 79 genes associated with SN, 35 SNPs in 28 genes associated with HD, 132 SNPs in 68 genes associated with GY, 89 SNPs in 49 genes associated with PN, 207 SNPs in 97 genes associated with TSN, 120 SNPs in 68 genes associated with FGN, 76 SNPs in 29 genes associated 

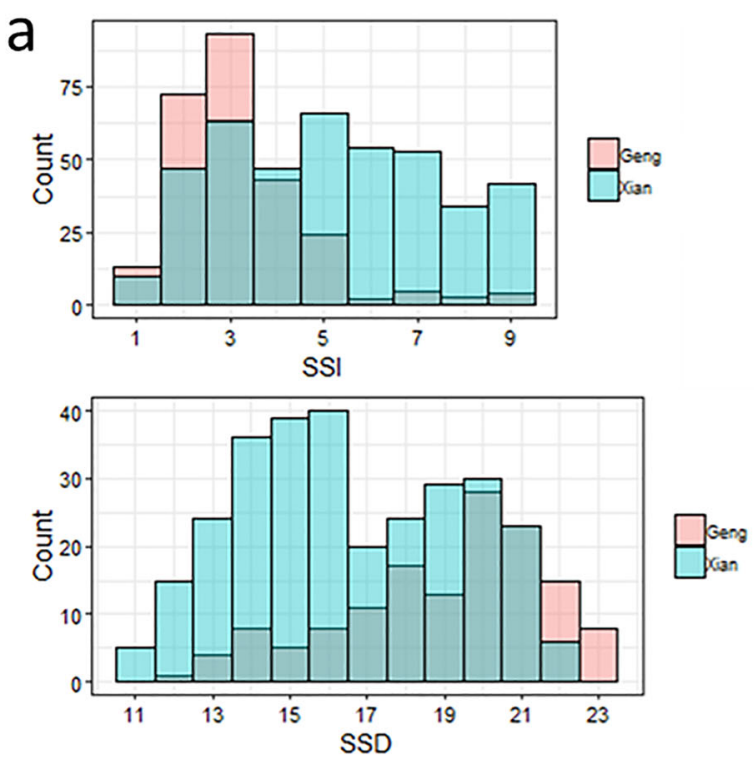

b

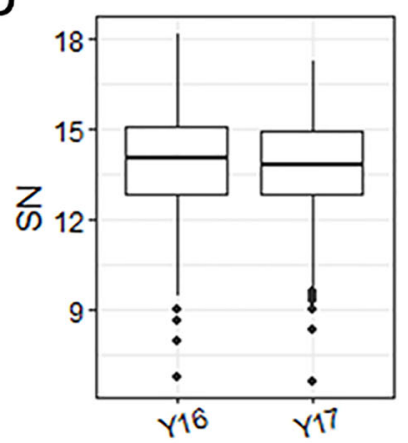

Environment

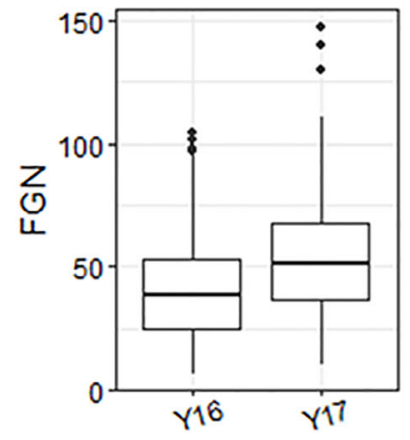

Environment

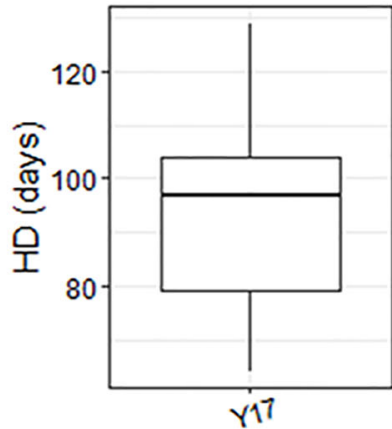

Environment

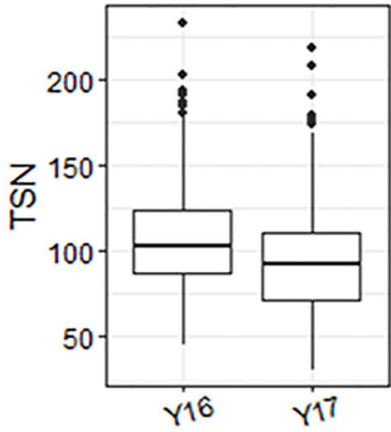

Environment

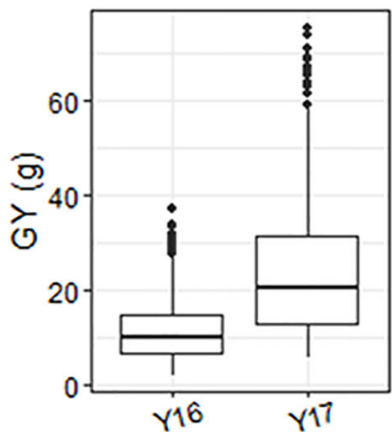

Environment

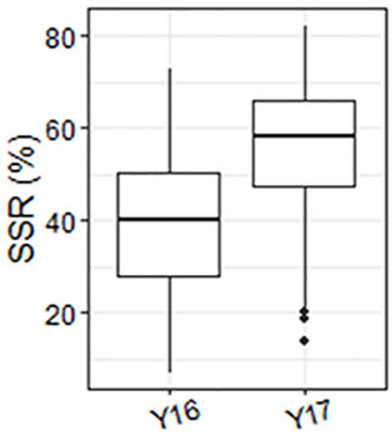

Environment

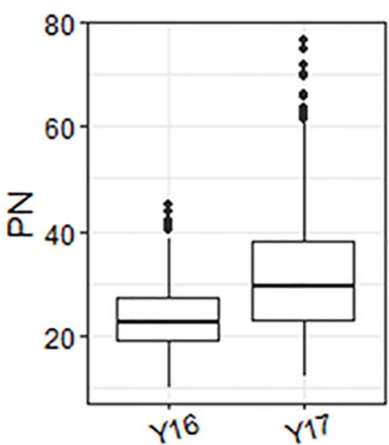

Environment

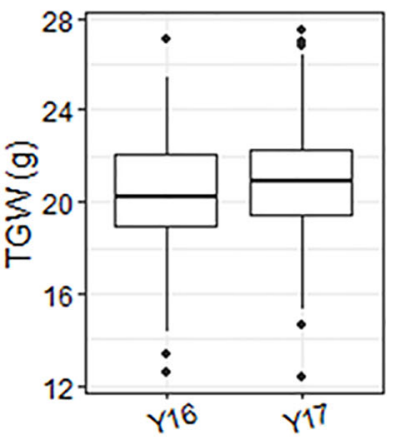

Environment

Fig. 1. Phenotypic distributions and correlations. (a) Distributions of SSI (salt stress injury) and SSD (seedling survival days) measured under hydroponic condition. "Count" indicated the number of rice accessions. Blue and red indicated Xian (Indica) and Geng (Japonica) subspecies, respectively. (b) Distributions of traits measured under saline field conditions. SN: seedling number; HD: heading date; GY: grain yield per plot; PN: total panicle number; FGN: filled grain number; TGW: thousand-grain weight; TSN: total spikelet number per plot; SSR: seed setting rate. (c) Pairwise correlations of traits measured under saline field conditions. The correlation coefficients ( $r$ ) multiplied by 100 on the lower triangle, upper triangle, and principal diagonal show the correlations in 2016, 2017, and between the two years, respectively. The areas and colors of the ellipses show the absolute values of the corresponding $r$. Right and left oblique ellipses indicate positive and negative correlations, respectively. The values without ellipses indicate that the correlations were not significant at $P<0.05$. 
Table 1 Variance components and heritability estimates for agronomic traits investigated under salt stress conditions in 2016 and 2017

\begin{tabular}{ccccccc}
\hline Trait & $V_{G}$ & $V_{G E I}$ & $V_{\text {Rep }}$ & \multicolumn{1}{c}{$V_{E}$} & $V_{e}$ & $h^{2}$ \\
\hline GY & 109.55 & 48.88 & 4.11 & 99.56 & 126.49 & 0.70 \\
FGN & 343.18 & 128.0 & 19.13 & 77.41 & 300.96 & 0.75 \\
TSN & 829.52 & 54.01 & 11.39 & 125.53 & 512.92 & 0.88 \\
SSR & 147.59 & 57.66 & 15.16 & 149.23 & 143.26 & 0.74 \\
TGW & 2.63 & 2.62 & 0.24 & 0.04 & 2.06 & 0.59 \\
PN & 90.69 & 9.85 & 6.04 & 55.18 & 113.21 & 0.80 \\
SN & 1.60 & 1.40 & 0.24 & 0.00 & 3.26 & 0.60 \\
\hline V
\end{tabular}

$V_{G}$ : genotype variance; $V_{G E l}$ : genotype by environment interaction variance; $V_{E}$ : environment variance; $V_{R e p}$ : replication variance within environment; $V_{e}$ : residual variance; $h^{2}$ : narrow-sense heritability. Trait abbreviations are as given in the legend to Figure 1.

with SSR, and four SNPs in three genes associated with TGW (Table 3 and Additional file 1: Table S2).

\section{Promising candidate genes for ST in rice}

In total, 230 genes in which expression is known to be responsive to salt stress were identified by searches of the funRiceGenes and QTARO databases (Additional file 1: Table S3). Among the 903 rice genes associated with the measured traits, 43 genes shared the same functional annotations and GO terms in the "biological process" and "molecular function" GO categories with the 230 known genes (Additional file 1: Table S4). These genes encode 21 types of putative proteins involving 35 different molecular functions and 45 biological processes (Fig. 3 and Table 4). We performed haplotype analyses and identified 2-6 major haplotypes (comprising more than 10 accessions) for these 43 genes (Fig. 4). The differences in target traits between/ among haplotypes were tested by ANOVA (Additional file 1: Table S5), and 15 significant genes were detected, including one gene for SSI, two genes for SSD, one gene for HD, two genes for GY, four genes for PN, two genes for FGN, one gene for SSR, and two genes for TSN (Table 4 and Fig. 4). Among them, LOC_ Os $04 g 51830$ and LOC_Os04g52140 were detected for PN in both 2016 and 2017, and LOC_Os05g31730 was detected for SSI and SSD. The above genes encompassed 10 different predicted functions, such as a MYB family transcription factor, protein kinase domain containing proteins, and transporter proteins (Table 4).

\section{Promising candidate genes associated with GY under saline conditions}

Among the 15 promising candidates, two genes (LOC_Os02g49700 and LOC_Os03g07480) detected in the Geng subgroup were especially attractive, because they were associated with GY and explained 32.7\% and $26.4 \%$ of the phenotypic variance (Table 4). LOC_Os02g49700 is predicted to encode a homeoboxassociated leucine zipper protein. One polymorphic locus, S1_30381323 (A/C), was identified in the 5' UTR of the gene in the Geng subgroup, and the A to $\mathrm{C}$ mutation could potentially increase GY significantly under saline stress conditions. For LOC_Os03g07480, six polymorphic SNPs were identified in the gene that that accounted for two major haplotypes. All of these SNPs are located in introns except S3_3800635 (T/C) that results in a synonymous mutation (Fig. 5). We

Table 2 Distribution of high quality SNP markers in the rice genome

\begin{tabular}{|c|c|c|c|c|c|c|c|}
\hline \multirow[t]{2}{*}{ Chr } & \multicolumn{2}{|l|}{ All SNPs ${ }^{a}$} & \multicolumn{2}{|l|}{$10 \%$ set $^{b}$} & \multicolumn{3}{|c|}{ SNPS within genes $^{c}$} \\
\hline & No. of SNPS & $\begin{array}{l}\text { Spacing } \\
\text { (bp) }\end{array}$ & No. of SNPS & $\begin{array}{l}\text { Spacing } \\
\text { (bp) }\end{array}$ & No. of genes & No. of SNPS & $\begin{array}{l}\text { Spacing } \\
\text { (bp) }\end{array}$ \\
\hline 1 & 404,439 & 107 & 40,444 & 1070 & 5468 & 128,891 & 336 \\
\hline 2 & 337,347 & 107 & 33,735 & 1065 & 4577 & 105,849 & 339 \\
\hline 3 & 311,509 & 117 & 31,151 & 1169 & 4962 & 94,538 & 385 \\
\hline 4 & 298,569 & 119 & 29,857 & 1189 & 3758 & 98,508 & 360 \\
\hline 5 & 240,251 & 125 & 24,026 & 1244 & 3812 & 73,139 & 409 \\
\hline 6 & 290,332 & 108 & 29,034 & 1073 & 3720 & 91,561 & 340 \\
\hline 7 & 275,472 & 108 & 27,548 & 1077 & 3482 & 88,151 & 336 \\
\hline 8 & 281,408 & 101 & 28,141 & 1010 & 3278 & 90,526 & 314 \\
\hline 9 & 212,314 & 108 & 21,232 & 1078 & 2656 & 67,287 & 340 \\
\hline 10 & 248,350 & 93 & 24,835 & 934 & 2724 & 77,376 & 299 \\
\hline 11 & 300,367 & 97 & 30,037 & 965 & 3088 & 102,704 & 282 \\
\hline 12 & 255,594 & 108 & 25,560 & 1077 & 2807 & 82,874 & 332 \\
\hline Total & $3,455,952$ & 108 & 345,600 & 1079 & 44,332 & $1,101,404$ & 339 \\
\hline
\end{tabular}

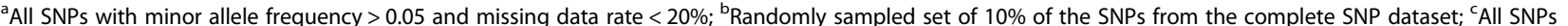
distributed in 44,332 annotated genes 
a
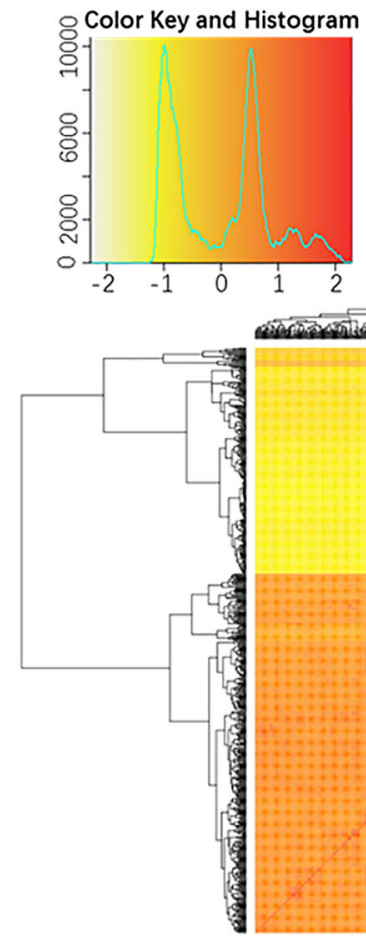

b

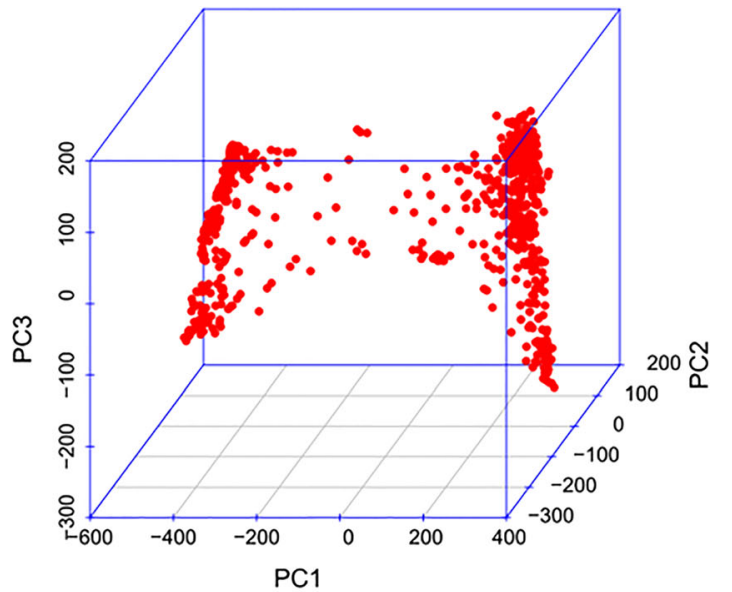

Fig. 2 Population structure of the rice germplasm association panel revealed by kinship (a) and principle component analysis (PCA) (b)

Table 3 Summary of the GWAS results for all 10 measured traits

\begin{tabular}{|c|c|c|c|c|c|c|c|c|c|c|c|c|c|}
\hline Condition & Group & Association & SSI & SSD & SN & $\mathrm{HD}$ & GY & PN & TSN & FGN & SSR & TGW & Total \\
\hline \multirow[t]{6}{*}{ Hydroponics } & \multirow[t]{2}{*}{ Geng } & No. SNPs & 83 & 25 & - & - & - & - & - & - & - & - & 107 \\
\hline & & No. SNPs & 56 & 13 & - & - & - & - & - & - & - & - & 68 \\
\hline & \multirow[t]{2}{*}{ Xian } & No. SNPs & 105 & 108 & - & - & - & - & - & - & - & - & 165 \\
\hline & & No. SNPs & 39 & 50 & - & - & - & - & - & - & - & - & 74 \\
\hline & \multirow[t]{2}{*}{ Total } & No. SNPs & 188 & 133 & - & - & - & - & - & - & - & - & 272 \\
\hline & & No. SNPs & 95 & 63 & - & - & - & - & - & - & - & - & 142 \\
\hline \multirow[t]{6}{*}{ Y16 } & \multirow[t]{2}{*}{ Geng } & No. SNPs & - & - & 12 & - & 6 & 129 & 7 & 6 & 9 & 0 & 169 \\
\hline & & No. SNPs & - & - & 11 & - & 5 & 44 & 7 & 5 & 2 & 0 & 74 \\
\hline & \multirow[t]{2}{*}{ Xian } & No. SNPs & - & - & 257 & - & 21 & 101 & 18 & 282 & 58 & 179 & 862 \\
\hline & & No. SNPs & - & - & 93 & - & 15 & 52 & 15 & 88 & 48 & 63 & 342 \\
\hline & \multirow[t]{2}{*}{ Total } & No. SNPs & - & - & 269 & - & 27 & 230 & 25 & 288 & 67 & 179 & 1031 \\
\hline & & No. SNPs & - & - & 104 & - & 20 & 96 & 22 & 93 & 50 & 63 & 415 \\
\hline \multirow[t]{6}{*}{ Y17 } & \multirow[t]{2}{*}{ Geng } & No. SNPs & - & - & 85 & 16 & 113 & 48 & 16 & 59 & 13 & 0 & 341 \\
\hline & & No. SNPs & - & - & 22 & 14 & 58 & 23 & 12 & 22 & 8 & 0 & 151 \\
\hline & \multirow[t]{2}{*}{ Xian } & No. SNPs & - & - & 101 & 19 & 19 & 41 & 191 & 61 & 63 & 4 & 492 \\
\hline & & No. SNPs & - & - & 57 & 14 & 10 & 26 & 85 & 46 & 21 & 3 & 256 \\
\hline & \multirow[t]{2}{*}{ Total } & No. SNPs & - & - & 186 & 35 & 132 & 89 & 207 & 120 & 76 & 4 & 832 \\
\hline & & No. SNPs & - & - & 79 & 28 & 68 & 49 & 97 & 68 & 29 & 3 & 403 \\
\hline
\end{tabular}




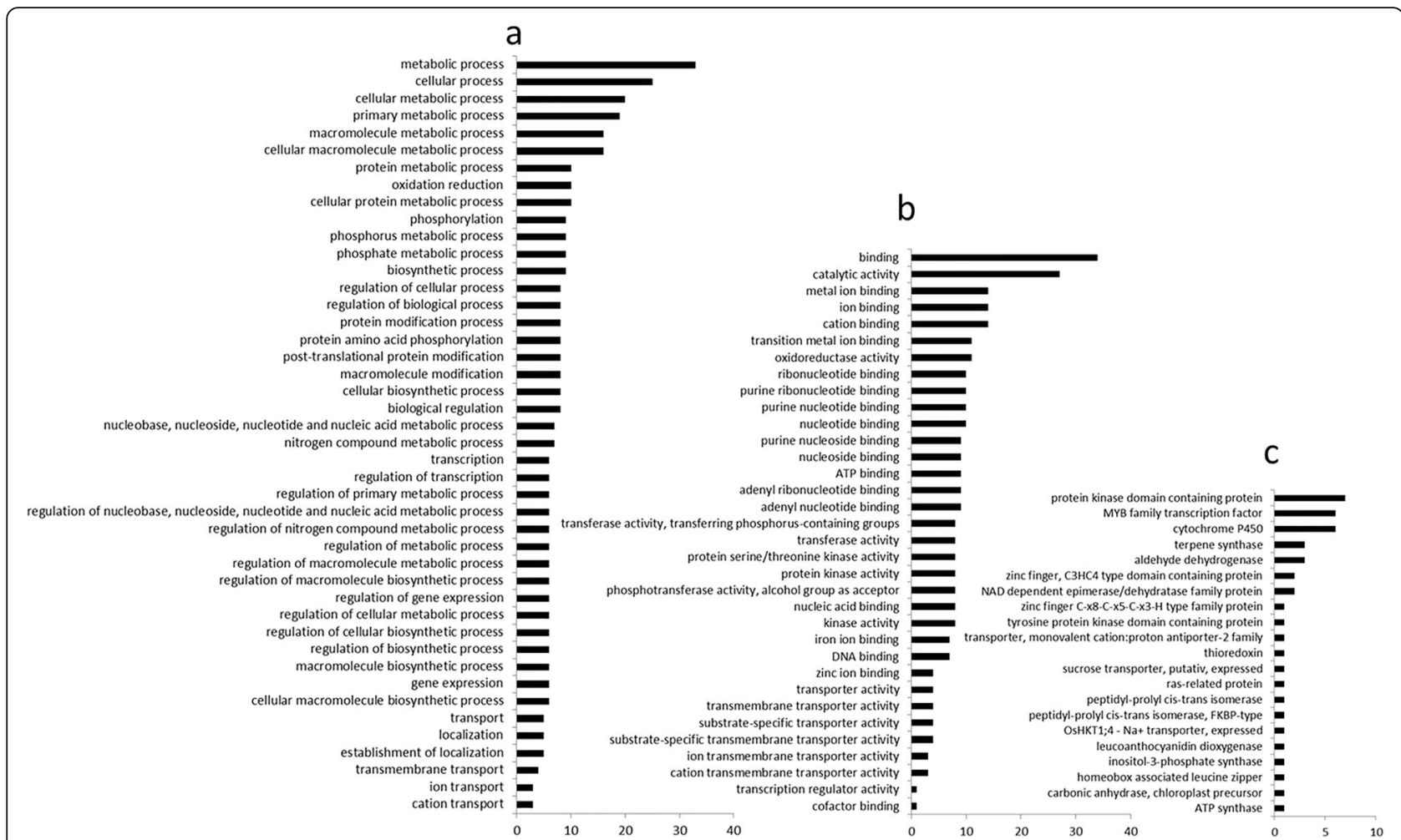

Fig. 3 The GO terms represented in the "biological process" (a) and "molecular function" (b) GO categories, and the putative encoded proteins (c) for the 43 identified candidate genes

further analyzed associations between haplotypes for the other 13 promising genes with GY, and found that another two genes, LOC_OsO3g28300 and LOC_ Os04g52140, associated with SSR and PN also affected GY and explained $11.5 \%$ and $7.7 \%$ of the phenotypic variance, respectively, in the Geng subgroup (Additional >file 1: Table S6). For $L O C_{-}$ Os03g28300, seven SNPs were identified that formed five major haplotypes. One SNP S3_16283872 (T/C) was located in an exon, resulting in a nonsynonymous mutation (Ser/Pro), while the other SNPs were in the UTRs and introns (Fig. 5). For LOC_Os04g52140, seven polymorphic loci were identified which formed three major haplotypes. All of these SNPs are located in the UTRs or introns except for S4_30974542 (C/T) which is present in an exon and causes an amino acid change from Thr to Met (Fig. 5).

\section{Discussion}

\section{GWAS using SNPs within genes}

GWAS has been widely used in rice for various studies that usually employ tens to hundreds of thousands of SNP markers distributed across the entire genome. Recently, the 3 K RGP sequenced a core collection of 3024 rice accessions and generated more than $32 \mathrm{M}$ SNP data, which provided us the opportunity to perform GWAS using higher density markers (Wang et al. 2018). Also, a GWAS using SNPs within genes allowed us to detect candidate genes directly and reduce computation time as well. In the present study, 3,455,952 high quality SNP markers (MAF $\geq 0.05$ and missing data rate $<20 \%$ ) were screened from the raw $32 \mathrm{M}$ SNPs. Among these, 1,101,404 SNPs within genes were further extracted to conduct the GWAS which could further reduce computation time dramatically. These SNPs are distributed in 44,332 annotated genes, and cover up to $80 \%$ of all annotated genes $(55,801)$ in the MSU Rice Genome Annotation Project Release 7 (http://rice.plantbiology.msu.edu/); this means that most of the genes, if not all, that contribute to the phenotypic variation in the measured traits could be detected. Our study provides a successful example for efficiently using SNP data from the $3 \mathrm{~K}$ RGP in a GWAS.

\section{Promising candidate genes for ST in rice}

At present, many genes related to rice ST have been identified by various methods such as map-based cloning, mutation, overexpression, and gene knockout, which allowed us to compare the association mapping results 
Table 4 Fifteen promising candidate genes for rice salt tolerance and their associations with the targeted traits identified by haplotype analysis

\begin{tabular}{|c|c|c|c|c|c|c|c|}
\hline \multirow[t]{2}{*}{ MSU.ID ${ }^{a}$} & \multirow[t]{2}{*}{ Function } & \multicolumn{2}{|l|}{ GWAS $^{\text {b }}$} & \multicolumn{2}{|l|}{ ANOVA $^{c}$} & \multirow[t]{2}{*}{ Trait $^{d}$} & \multirow[t]{2}{*}{ Env ${ }^{e}$} \\
\hline & & $P$ value & $R^{2}(\%)$ & P value & $\overline{R^{2}(\%)}$ & & \\
\hline Os09g28910 & carbonic anhydrase, chloroplast precursor & $6.60 \mathrm{E}-05$ & 3.5 & 0.0007 & 5.3 & TSN & Y17 \\
\hline Os02g49700 & homeobox associated leucine zipper & $5.20 \mathrm{E}-07$ & 11.8 & 1.10E-18 & 32.7 & GY & Y17 \\
\hline Os06g42130 & leucoanthocyanidin dioxygenase & $7.90 \mathrm{E}-05$ & 5.2 & 1.30E-05 & 8.1 & SSD & Hydroponics \\
\hline Os01g18240 & MYB family transcription factor & 4.00E-05 & 5.1 & $1.50 \mathrm{E}-07$ & 8.6 & PN & Y16 \\
\hline Os01g59660 & MYB family transcription factor & 7.70E-05 & 3.5 & 4.10E-07 & 10.6 & TSN & Y17 \\
\hline Os08g06240 & MYB family transcription factor & $8.30 \mathrm{E}-05$ & 4.4 & 4.70E-15 & 24.6 & $H D$ & Y17 \\
\hline Os04g51830 & OsHKT1;4 - Na + transporter & $1.60 \mathrm{E}-05$ & 10.8 & $1.90 \mathrm{E}-05$ & 14.1 & PN & Y16 \\
\hline Os04g51830 & OsHKT1;4 - Na + transporter & $1.80 \mathrm{E}-05$ & 7.6 & $2.20 \mathrm{E}-05$ & 10.9 & PN & Y17 \\
\hline Os01g18210 & peptidyl-prolyl cis-trans isomerase, FKBP-type & 6.30E-05 & 4.8 & $9.10 \mathrm{E}-06$ & 6.3 & PN & Y16 \\
\hline Os03g28300 & protein kinase domain containing protein & $1.40 \mathrm{E}-05$ & 6 & $3.90 \mathrm{E}-06$ & 10.6 & SSR & Y16 \\
\hline Os04g52140 & protein kinase domain containing protein & 4.70E-05 & 9.6 & $1.40 \mathrm{E}-06$ & 13.7 & PN & Y16 \\
\hline Os04g52140 & protein kinase domain containing protein & 9.70E-05 & 6.2 & 2.00E-07 & 13.3 & PN & Y17 \\
\hline Os04g56120 & protein kinase domain containing protein & $8.80 \mathrm{E}-05$ & 3.8 & $2.40 \mathrm{E}-08$ & 9.7 & FGN & Y17 \\
\hline Os03g07480 & sucrose transporter & 3.10E-05 & 8 & $6.40 \mathrm{E}-14$ & 26.4 & GY & Y17 \\
\hline Os01g23530 & terpene synthase & $5.30 \mathrm{E}-05$ & 3.7 & 0.0004 & 7.1 & SN & Y17 \\
\hline Os05g31730 & transporter, monovalent cation:proton antiporter-2 family & $2.10 \mathrm{E}-07$ & 6.2 & $5.40 \mathrm{E}-06$ & 9.7 & SSI & Hydroponics \\
\hline Os05g31730 & transporter, monovalent cation:proton antiporter-2 family & $2.00 \mathrm{E}-05$ & 6.1 & 2.90E-05 & 12.2 & SSD & Hydroponics \\
\hline Os01g06590 & zinc finger, C3HC4 type domain containing protein & $8.20 \mathrm{E}-05$ & 7.2 & 4.90E-06 & 13.4 & FGN & Y17 \\
\hline
\end{tabular}

${ }^{a}$ Gene names from the MSU Rice Genome Annotation Project Database; ${ }^{b}$ GWAS statistics for peak SNP within the gene; ${ }^{c}$ ANOVA for haplotypes of the gene with target traits; ${ }^{d}$ For trait name abbreviations see Fig.

from the present study with previously identified genes. Among the 903 genes associated with SSI and SSD at the seedling stage, and yield and its related traits measured under saline field conditions in Y16 and Y17, 43 candidate genes were identified that shared the same functional annotations and the same GO terms in the "biological process" and "molecular function" GO categories. Of these, 15 genes were further investigated by haplotype analysis and ANOVA. These genes are considered to be potential candidates to improve ST in rice.

Three genes predicted to encode protein kinase domain-containing proteins were detected; $L O C_{-}$ Os03g28300, LOC_Os04g52140, and LOC_Os04g56120. LOC_Os04g52140 (OsCTR3) is predicted to be a Raf-like Ser/Thr protein kinase CTR (constitutive tripleresponse)-like gene related to ethylene signaling (Wang et al. 2013). Its function with respect to ST in rice has not been reported, but ethylene signaling could modulate the salt response at different levels, including membrane receptors, components in the cytoplasm, and nuclear transcription factors in the pathway (Cao et al. 2008). Here, we identified its association with PN under salt stress conditions. The other two genes are novel genes that have not been reported previously.

Genes encoding MYB-type transcription factors have been shown to play important roles in abiotic stresses in many plant species, including drought and salinity stresses in rice; examples are OsMYB2 (Yang et al. 2012), OsJAmyb (Yokotani et al. 2013), OsMYB48-1 (Xiong et al. 2014), and OsMYB91 (Zhu et al. 2015). In the present study, three MYB family transcription factor genes were identified, including LOC_Os01g18240, LOC_Os01g59660, and LOC_Os08g06240. Among these, $L O C \_O s 01 g 18240$, also known as OsMYB61, regulates cellulose synthase genes (CESAs) in rice (Huang et al. 2015), and LOC_Os01g59660 (OsMYBGA) functions in the induction of gibberellin (GA)-dependent $\alpha$-amylase in the aleurone and floral organ and pollen development in rice (Kaneko et al. 2004), but the roles these genes play in rice ST was not characterized. The other MYB family transcription factor gene, $L O C_{-}$ Os08g06240, that is potentially related to salt stress was first identified here.

LOC_Os02g49700, a gene that is associated with GY, encodes a homeobox-associated leucine zipper protein and is the same as the two known genes OsHOX24 and Oshox22. Overexpression of OsHOX24 enhanced susceptibility to abiotic stresses in transgenic rice by modulating stress-responsive gene expression. The expression of Oshox 22 is strongly induced by salt stress. Rice plants homozygous for a T-DNA insertion in the promoter region of Oshox22 showed reduced Oshox22 expression 


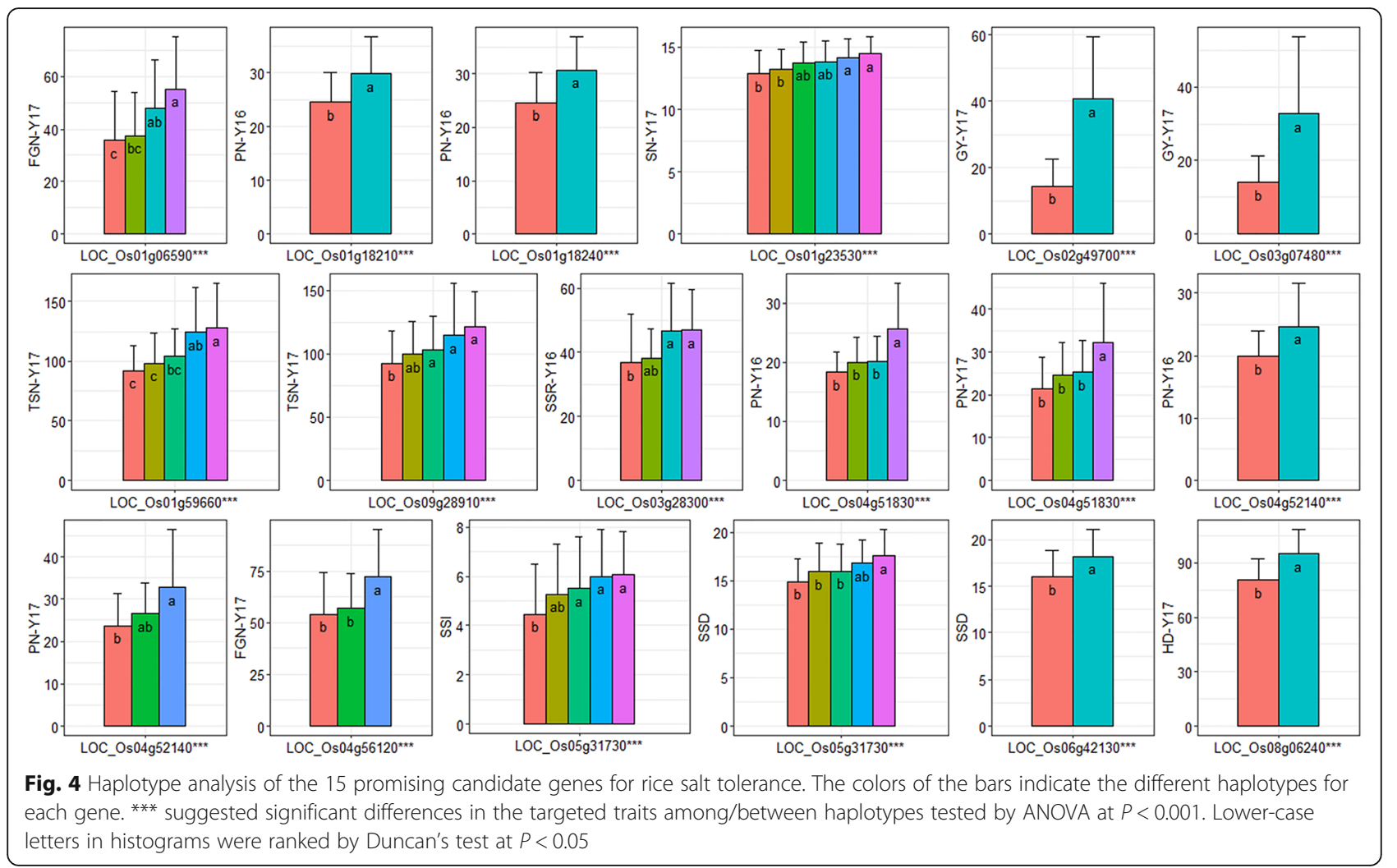

and enhanced tolerance to drought and salt stress at the seedling stage. In contrast, transgenic rice plants overexpressing Oshox22 showed decreased tolerance to drought and salt (Zhang et al. 2012). The SNPs present in the $5^{\prime}$ UTR of the gene may regulate its expression levels, which could result in increased GY under salt stress conditions.

LOC_Os03g07480 is a sucrose transporter gene also known as OsSUT1. OsSUT1 plays a role in carbon partitioning, specifically in grain filling and seed germination, and anti-sense lines in which this gene is silenced shown increased tolerance to salt stress (Siahpoosh et al. 2012). An association of LOC_OsO3g07480 with GY under saline stress conditions was identified in our study. We detected five mutations in introns and one synonymous mutation in an exon in this gene; thus, we can speculate that alternative splicing could result from these mutations and possibly alter the gene's function.

We identified LOC_Os01g06590, a gene encoding a zinc finger, C3HC4-type domain-containing protein that is associated with FGN. The functional annotation was the same as that of two previously-identified ST-related
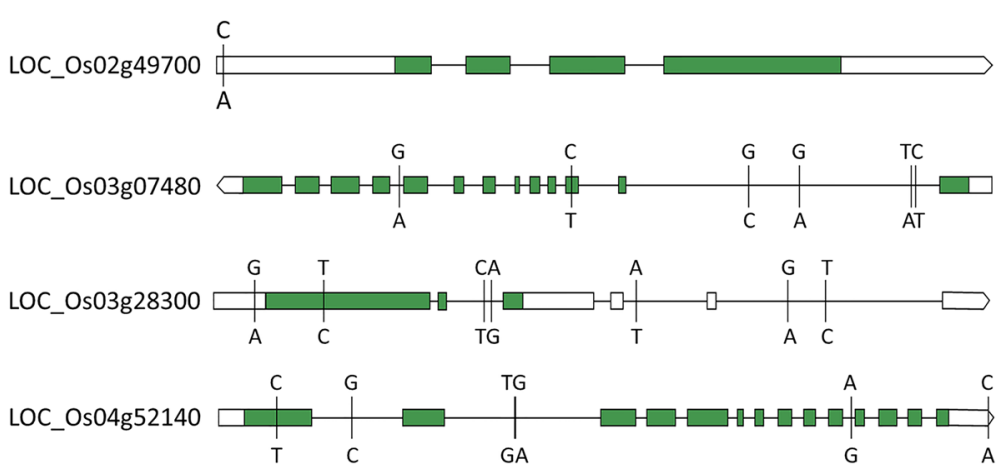

Fig. 5 Predicted structures of the four genes significantly associated with GY and the positions of the SNPs used for haplotype analysis. The green rectangles indicated exons 
genes, Osdsg1 and OsMAR1; the proteins encoded by these genes have been shown to possess E3 ubiquitin ligase activity (Park et al. 2010; Park et al. 2017).

The gene LOC_Os01g18210 associated with PN encodes a peptidyl-prolyl cis-trans isomerase, the same as two known ST-related genes OsCYP2 and OsCYP21-4 that encode cyclophilin proteins. OsCYP2 acts as a key regulator that controls ROS levels by modulating the activities of antioxidant enzymes at the level of translation, and expression of OsCYP2 is induced by salt stress (Ruan et al. 2011). Transgenic plants overexpressing OsCYP21-4 exhibited increased tolerance to salinity, possibly via regulation of peroxidase activity (Lee et al. 2015).

We found that LOC_Os01g23530 was significantly associated with $\mathrm{SN}$. Because the seeds were directly sown in the normal paddy field, the number of surviving seedlings observed one month after salinization from the two-leaf stage could reflect the salt tolerance at the seedling stage. $L O C_{-}$ Os01g23530 encodes a terpene synthase that is the same as the known ST-related gene OsKS2. OsKD2 is an ent-kaurene synthase that participates in GA biosynthesis and plays a significant role in the growth and development of higher plants. Moreover, OsKS2 transcripts are strongly induced by salinity and drought, which suggests that its expression might be related to abiotic stress defense in rice (Ji et al. 2014).

LOC_Os04g51830, also known as OsHKT1;4, encodes a $\mathrm{Na}^{+}$transporter. Certain class I transporters of the highaffinity $\mathrm{K}^{+}$transporter (HKT) family have been demonstrated to mediate leaf-blade $\mathrm{Na}^{+}$exclusion during salt stress via $\mathrm{Na}^{+}$-selective transport. Suzuki et al. (2016) reported that OsHKT1;4-mediated $\mathrm{Na}^{+}$transport in stems contributes to $\mathrm{Na}^{+}$exclusion from rice leaf blades at the reproductive growth stage under salt stress. We detected the association of LOC_Os04g51830 with PN (Table 4).

LOC_Os05g31730, a gene associated with SSI and SSD (Table 4), encodes a transporter belonging to the monovalent cation:proton antiporter-2 family and is the same as two known genes, OsNHX1 and OsNHX5. Proteins encoded by members of this gene family play important roles in the compartmentalization of $\mathrm{Na}^{+}$and $\mathrm{K}^{+}$that accumulate in the cytoplasm into vacuoles, and thus could improve rice ST (Fukuda et al. 2011). LOC_Os06g42130 encodes leucoanthocyanidin dioxygenase, which is the same as OsAns, a rice flavonoid pathway gene that is induced by high salt treatment (Ithal and Reddy 2004). LOC_Os06g42130 was found to be associated with SSD. LOC_Os09g28910 is associated with TSN and encodes carbonic anhydrase (CA), and the chloroplast precursor is same as that encoded by OsCA1. The expression of OsCA1 in rice leaves and roots is induced by salt stress (Yu et al. 2007).

\section{Application in breeding rice for ST}

Rice plants may be subject to salt stress at any development stage and even over the entire growth period.
Improving yield under saline stress conditions is the final goal of rice ST breeding. Because the correlation between the physiological indicators at the seedling stage and final yield at the reproductive stage under salt stress is poor (Hossain et al., 2015), we also found that rice plants that are salt tolerant as seedlings might not necessarily be high yielding when grown in a salinized paddy field, indicating that the genetic basis of rice ST at the seedling and reproductive stages is different. Interestingly, in the current study, we still identified 10 genes associated with both ST-related traits (SSI, SSD, and/or SN) at the seedling stage and yield and its related traits (GY, PN, TSN, FGN, SSR, and/or TGW) (Additional file 1: Table S2), which might be due to pleiotropism or linkage disequilibrium. Thus, pyramiding genes that function at different developmental stages or all stages will facilitate molecular breeding for ST in rice. Of the 15 promising genes for rice ST identified here, four genes (LOC_OsO2g49700, LOC_Os03g28300, OsCTR3, and OsSUT1) were found to be significantly associated with GY and its related traits (SSR and PN) under saline conditions, while two genes ( $L O C_{-}$ Os05g31730 and LOC_OsO1g23530) were significantly associated with SSI, SSD, and SN at the seedling stage. Of course, further studies are needed to validate the functions of these genes, possibly by overexpression in transgenic plants or by CRISPR/Cas genome editing. Even these candidates are not causal genes, but the SNPs in the gene sequences are also suitable for marker-assisted breeding because of the high degree of linkage disequilibrium between them. Thus, after converting these linked SNPs into Kompetitive Allele Specific PCR (KASP) markers, markerassisted breeding could be carried out to improve seedling development and yield production by deploying different yield and yield-related ST genes with seedling-stage-ST genes under saline field conditions. We identified seven accessions, including CX315, IRIS 313-11,571, IRIS 313-11,584, IRIS 313-11,652, IRIS 313-11,698, IRIS 313-9724, and IRIS 313-9725 that carry favorable haplotypes of the four genes ( $L O C_{-}$ Os02g49700, LOC_Os03g07480, LOC_Os03g28300, and LOC_Os04g52140) that have high GY under saline stress conditions, and three accessions, including B255, CX250, and IRIS 313-10,275, that carry favorable haplotypes of two genes (LOC_OsO1g23530 and LOC_Os05g31730) with good ST at the seedling stage (Additional file 1: Table S7); all of these lines could be used as parents in rice ST breeding.

\section{Conclusion}

ST characters varied widely in the rice association panel at both the seedling and reproductive stages under salt 
stress conditions. A GWAS utilizing high density SNPs within genes followed by functional annotation, GO analysis, and haplotype analysis was effective at detected candidate genes related to the investigated traits. We identified a total of 15 promising gene candidates, of which two are known to be involved in ST, three were previously cloned in relation to other traits but their roles in rice ST were not characterized, and another 10 novel genes that are potential candidates for rice ST. Seven high yield rice accessions carrying favorable haplotypes for the four genes significantly associated with GY under the saline stress conditions and three accessions with favorable haplotypes for the two genes with good ST at the seedling stage were identified. These promising candidate genes that we identified as being associated with rice ST are valuable resources for future functional characterization and marker-assisted breeding to improve rice grain yield and production in salinized paddy fields.

\section{Supplementary information}

Supplementary information accompanies this paper at https://doi.org/10. 1186/s12284-019-0349-z.

Additional file 1: Table S1. Rice accessions included in the ST association panel. Table S2. All identified marker-trait associations and the corresponding annotated genes. Table S3. Known genes involved in rice salt stress recorded in the QTARO and funricegenes databases. Table S4. The promising candidate genes for ST showed the same functional annotations and GO terms as genes known to be involved in salt stress. Table S5. ANOVA for haplotypes of 43 genes with the target traits. Table S6. ANOVA for haplotypes of the 15 promising genes with GY in each subgroup grown in two environments. Table S7. Well performing accessions carrying favorable haplotypes of four genes associated with GY and two genes associated with SSI and SSD

Additional file 2: Figure S1A-I. Manhattan and Q-Q plots of the GWAS for all measured traits

\begin{abstract}
Abbreviations
3 K RGP: 3 K Rice genome project; ANOV: Analysis of variance.; FGN: filled grain number.; GAPIT: Genome Association and Prediction Integrated Tool.; GO: gene ontology.; GWAS: Genome-wide Association Study.; GY: grain yield per plot.; HD: heading date.; MAF: minor allele frequency.; QTL: quantitative trait loci.; SNP: single nucleotide polymorphism.; SSD: seedling survival days.; SSI: salt stress injury.; SSR: seed setting rate.; ST: salt tolerance.; SN: seedling number.; TGW: thousand-grain weight.; TPN: total panicle number.; TSN: total spikelet number per plot.
\end{abstract}

\section{Acknowledgements}

Not applicable.

\section{Authors' contributions}

$J \mathrm{LX}$ designed and supervised the research. CL, KC, XQZ, CCS, and MLD performed phenotyping. XJQ and YLP analyzed data. XQW, YJZ, RWY, and DYX contributed reagents/materials/analysis tools. YLP and JLX wrote the paper. All authors read and approved the final manuscript.

\section{Funding}

This project was funded by the National Key Technology Support Program (2015BAD02B01-2-1), the National Natural Science Foundation of China (31471429), the Fundamental Research Funds for Science, Technology and Innovation Commission of Shenzhen Municipality (JCYJ20160530191619099), the Agricultural Science and Technology Innovation Program and the
Cooperation and Innovation Mission (CAAS-ZDXT201800), and Guangdong Provincial Science and Technology Project (2017B020201003).

\section{Availability of data and materials}

The datasets used and/or analyzed during the current study are available from the corresponding author upon reasonable request.

Ethics approval and consent to participate

Not applicable.

\section{Consent for publication}

Not applicable.

\section{Competing interests}

The authors declare that they have no competing interests.

\section{Author details}

${ }^{1}$ College of Agriculture, Yangtze University, Jingzhou 434025, China.

'Laboratory of Lingnan Modern Agriculture/ Agricultural Genomics Institute at Shenzhen, Shenzhen 518120, China. ${ }^{3}$ Institute of Crop Sciences, Chinese Academy of Agricultural Sciences, Beijing 100081, China. ${ }^{4}$ State Key Laboratory of Crop Biology, College of Agronomy, Shandong Agricultural University, Taian 271018, China.

Received: 30 July 2019 Accepted: 8 November 2019

Published online: 02 December 2019

\section{References}

Abdullah Z, Khan MA, Flowers TJ (2001) Causes of sterility in seed set of rice under salinity stress. J Agron Crop Sci 187:25-32

Alexandrov N, Tai S, Wang W, Mansueto L, Palis K, Fuentes RR, Ulat VJ, Chebotarov D, Zhang G, Li Z, Mauleon R, Hamilton RS, McNally KL (2015) SNP-seek database of SNPs derived from 3000 rice genomes. Nucleic Acids Res 43:D1023-D1027

Blumwald E, Grover A (2006) Salt tolerance. In: Halford NG (ed) Plant biotechnology. John Wiley \& Sons, Ltd, pp 206-224

Cao YR, Chen SY, Zhang JS (2008) Ethylene signaling regulates salt stress response: An overview. Plant Signal Behav 3:761-763

Chai L, Zhang J, Pan XB, Zhang F, Zheng TQ, Zhao XQ, Wang WS, Ali J, Xu JL, Li ZK (2013) Advanced backcross QTL analysis for the whole plant growth duration salt tolerance in rice (Oryza sativa L.). J Integr Agric 13:1609-1620

Cheng J, He Y, Yang B, Lai Y, Wang Z, Zhang H (2015) Association mapping of seed germination and seedling growth at three conditions in indica rice (Oryza sativa L.). Euphytica 206:1-13

Cheng L, Wang Y, Meng L, Hu X, Cui Y, Sun Y, Zhu L, Ali J, Xu J, Li Z (2011) Identification of salt-tolerant QTLs with strong genetic background effect using two sets of reciprocal introgression lines in rice. Genome 55:45-55

Fageria NK (1985) Salt tolerance of rice cultivars. Plant Soil 88:237-243

Fukuda A, Nakamura A, Hara N, Toki S, Tanaka Y (2011) Molecular and functional analyses of rice $\mathrm{NHX}$-type $\mathrm{Na}^{+} / \mathrm{H}^{+}$antiporter genes. Planta 233:175-188

Gregorio GB, Senadhira D, Mendoza RD, Manigbas NL, Roxas JP, Guerta CQ (2002) Progress in breeding for salinity tolerance and associated abiotic stresses in rice. Field Crops Res 76:91-101

Harrell, FE Jr (2015) Hmisc: Harrell Miscellaneous. R package version 3.16-0 Hossain H, Rahman MA, Alam MS, Singh RK (2015) Mapping of quantitative trait loci associated with reproductive-stage salt tolerance in rice. J Agron Crop Sci 201:17-31

Hu S, Tao H, Qian Q, Guo L (2012) Genetics and molecular breeding for salttolerance in rice. Rice Genomics and Genetics 3:39-49

Huang D, Wang S, Zhang B, Shang-Guan K, Shi Y, Zhang D, Liu X, Wu K, Xu Z, Fu $X$, Zhou Y (2015) A gibberellin-mediated DELLA-NAC signaling cascade regulates cellulose synthesis in rice. Plant Cell 27:1681-1696

IRRI (2013) Standard evaluation system (SES) for Rice 5th edn. IRRI, P.O. Box 933 1099 Manila, Philippines

Ismail A, Thomson M (2011) Molecular breeding of Rice for problem soils. In: Varshney RK (ed) Costa de Oliveira A. Root Genomics. Springer, Berlin Heidelberg, pp 289-311

Ithal N, Reddy AR (2004) Rice flavonoid pathway genes, OsDfr and OsAns, are induced by dehydration, high salt and $\mathrm{ABA}$, and contain stress responsive promoter elements that interact with the transcription activator, OSC1-MYB. Plant Sci 166:1505-1513 
Ji SH, Gururani MA, Lee JW, Ahn BO, Chun SC (2014) Isolation and characterisation of a dwarf rice mutant exhibiting defective gibberellins biosynthesis. Plant Biol 16:428-439

Kaneko M, Inukai Y, Ueguchi-Tanaka M, Itoh H, Izawa T, Kobayashi Y, Hattori T, Miyao A, Hirochika H, Ashikari M, Matsuoka M (2004) Loss-of-function mutations of the rice GAMYB gene impair a-amylase expression in aleurone and flower development. Plant Cell 16:33-44

Kumar V, Singh A, Mithra SV, Krishnamurthy SL, Parida SK, Jain S, Tiwari KK, Kumar P, Rao AR, Sharma SK, Khurana JP, Singh NK, Mohapatra T (2015) Genomewide association mapping of salinity tolerance in rice (Oryza sativa). DNA Res 22:133-145

Lee SS, Park HJ, Jung WY, Lee A, Yoon DH, You YN, Kim HS, Kim BG, Ahn JC, Cho HS (2015) OsCYP21-4, a novel Golgi-resident cyclophilin, increases oxidative stress tolerance in rice. Front Plant Sci 6:797-797

Molla KA, Debnath AB, Ganie SA, Mondal TK (2015) Identification and analysis of novel salt responsive candidate gene based SSRs (cgSSRs) from rice (Oryza sativa L.). BMC Plant Biol 15:122-132

Park GG, Park JJ, Yoon J, Yu SN, An G (2010) A RING finger E3 ligase gene, Oryza sativa delayed seed germination 1 (OsDSG1), controls seed germination and stress responses in rice. Plant Mol Biol 74:467-478

Park YC, Chapagain S, Seong Jang C (2017) The microtubule-associated RING finger protein 1 (OsMAR1) acts as a negative regulator for salt-stress response through the regulation of OCPI2 (O. sativa chymotrypsin protease inhibitor 2). Planta 247:875-886

Ren ZH, Gao JP, Li LG, Cai XL, Huang W, Chao DY, Zhu MZ, Wang ZY, Luan S, Lin HX (2005) A rice quantitative trait locus for salt tolerance encodes a sodium transporter. Nat Genet 37:1141-1146

Ruan SL, Ma HS, Wang SH, Fu YP, Xin Y, Liu WZ, Wang F, Tong JX, Wang SZ, Chen HZ (2011) Proteomic identification of OSCYP2, a rice cyclophilin that confers salt tolerance in rice (Oryza sativa L.) seedlings when overexpressed. BMC Plant Biol 11:34-34

Siahpoosh MR, Sanchez DH, Schlereth A, Scofield GN, Furbank RT, van Dongen JT, Kopka J (2012) Modification of OsSUT1 gene expression modulates the salt response of rice Oryza sativa cv. Taipei 309. Plant Sci 182:101-111

Suzuki K, Yamaji N, Costa A, Okuma E, F, Katsuhara M, Wang C, Tanoi K, Murata Y, I. Schroeder J, Feng Ma J (2016) OsHKT1;4-mediated $\mathrm{Na}^{+}$transport in stems contributes to $\mathrm{Na}^{+}$exclusion from leaf blades of rice at the reproductive growth stage upon salt stress. BMC Plant Biol 16

Wang Q, Zhang W, Yin Z, Wen CK (2013) Rice CONSTITUTIVE TRIPLE-RESPONSE2 is involved in the ethylene-receptor signalling and regulation of various aspects of rice growth and development. J Exp Bot 64:4863-4875

Wang W, Mauleon R, Hu Z, Chebotarov D, Tai S, Wu Z, Li M, Zheng T, Fuentes RR, Zhang F, Mansueto L, Copetti D, Sanciangco M, Palis KC, Xu J, Sun C, Fu B, Zhang H, Gao Y, Zhao X, Shen F, Cui X, Yu H, Li Z, Chen M, Detras J, Zhou Y, Zhang X, Zhao Y, Kudrna D, Wang C, Li R, Jia B, Lu J, He X, Dong Z, Xu J, Li Y, Wang M, Shi J, Li J, Zhang D, Lee S, Hu W, Poliakov A, Dubchak I, Ulat VJ, Borja FN, Mendoza JR, Ali J, Li J, Gao Q, Niu Y, Yue Z, Naredo MEB, Talag J, Wang X, Li J, Fang X, Yin Y, Glaszmann J-C, Zhang J, Li J, Hamilton RS, Wing RA, Ruan J, Zhang G, Wei C, Alexandrov N, McNally KL, Li Z, Leung H (2018) Genomic variation in 3,010 diverse accessions of Asian cultivated rice. Nature 557:43-49

Wang Z, Cheng J, Chen Z, Huang J, Bao Y, Wang J, Zhang H (2012) Identification of QTLs with main, epistatic and QTL x environment interaction effects for salt tolerance in rice seedlings under different salinity conditions. Theoret Appl Genetics 125:807-815

Wang Z, Wang J, Bao Y, Wu Y, Zhang H (2011) Quantitative trait loci controlling rice seed germination under salt stress. Euphytica 178:297-307

Xiong H, Li J, Liu P, Duan J, Zhao Y, Guo X, Li Y, Zhang H, Ali J, Li Z (2014) Overexpression of OSMYB48-1, a novel MYB-related transcription factor, enhances drought and salinity tolerance in rice. PLoS One 9:e92913-e92913

Yang A, Dai XY, Zhang WH (2012) A R2R3-type MYB gene, OSMYB2, is involved in salt, cold, and dehydration tolerance in rice. J Exp Bot 63:2541-2556

Yeo AR, Yeo ME, Flowers SA, Flowers TJ (1990) Screening of rice (Oryza sativa L.) genotypes for physiological characters contributing to salinity resistance, and their relationship to overall performance. Theoret Appl Genetics 79:377-384

Yokotani N, Ichikawa T, Kondou Y, Iwabuchi M, Matsui M, Hirochika H, Oda K (2013) Role of the rice transcription factor JAmyb in abiotic stress response. J Plant Res 126:131-139

Yoshida S, Forno D, Cock J, Gomez K (1976) Laboratory manual for physiological studies of Rice. International Rice Research Institute (IRRI), Manila, Philippines
Yu S, Zhang X, Guan Q, Takano T, Liu S (2007) Expression of a carbonic anhydrase gene is induced by environmental stresses in Rice (Oryza sativa L.). Biotechnol Lett 29:89-94

Zhang S, Haider I, Kohlen W, Jiang L, Bouwmeester H, Meijer A, Schluepmann $H_{\text {, }}$ Liu CM, B F Ouwerkerk P (2012) Function of the HD-Zip I gene OsHOX22 in ABA-mediated drought and salt tolerances in rice. Plant Mol Biol 80

Zheng H, Wang J, Zhao H, Liu H, Sun J, Guo L, Zou D (2015) Genetic structure, linkage disequilibrium and association mapping of salt tolerance in japonica rice germplasm at the seedling stage. Mol Breed 35:1-16

Zhu N, Cheng SF, Liu XY, Du H, Dai MQ, Zhou DX, Yang WJ, Zhao Y (2015) The R2R3-type MYB gene OsMYB91 has a function in coordinating plant growth and salt stress tolerance in rice. Plant Sci 236:146-156

\section{Publisher's Note}

Springer Nature remains neutral with regard to jurisdictional claims in published maps and institutional affiliations.

\section{Submit your manuscript to a SpringerOpen ${ }^{\circ}$ journal and benefit from:}

- Convenient online submission

- Rigorous peer review

- Open access: articles freely available online

- High visibility within the field

- Retaining the copyright to your article

Submit your next manuscript at $\boldsymbol{\nabla}$ springeropen.com 